\title{
TRANSPORT AND MAGNETIC PROPERTIES OF PbTe:Cr AND PbSnTe:Cr*
}

\author{
T. STory, E. Grodzicka, B. Witkowska, J. Górecka \\ AND W. DoBrowolsKI \\ Institute of Physics, Polish Academy of Sciences \\ Al. Lotników 32/46, 02-668 Warszawa, Poland
}

\begin{abstract}
We present the results of the experimental studies of the low temperature transport and magnetic properties of $\mathrm{PbTe}, \mathrm{Pb}_{1-x} \mathrm{Sn}_{x} \mathrm{Te}(x \leq 0.3)$ and SnTe crystals doped with 0.5 at.\% of chromium. Cr was found to be a resonant donor in $\mathrm{PbTe}$ and $\mathrm{PbSnTe}$. Magnetic susceptibility measurements revealed that $\mathrm{PbTe}: \mathrm{Cr}$ and $\mathrm{Pb}_{1-x} \mathrm{Sn}_{x} \mathrm{Te}: \mathrm{Cr}(x \leq 0.2)$ are Curie paramagnets whereas SnTe:Cr exhibits van Vleck paramagnetism.
\end{abstract}

PACS numbers: 75.50.Pp, 72.20.My

In this communication we present the results of the experimental studies of the crystals of $\mathrm{PbTe}: \mathrm{Cr}, \mathrm{Pb}_{1-x} \mathrm{Sn}_{x} \mathrm{Te}: \mathrm{Cr}$ and $\mathrm{SnTe}: \mathrm{Cr}$. Our interest in these new semimagnetic semiconductors (SMSC) is threefold. Firstly, this can be an important extension of the family of IV-VI SMSC to the systems with nonzero orbital magnetic moment of transition metal impurities. Secondly, it is an interesting resonant donor (acceptor) system with matrix composition controlled energetic location of the impurity level. Finally, the doping with $\mathrm{Cr}$ offers new possibilities in controlling the electric properties of IV-VI semiconductors.

All the studied samples were grown by the Bridgman method. We investigated the set of samples of $\mathrm{Pb}_{1-x} \mathrm{Sn}_{x} \mathrm{Te}$ covering the composition range $x \leq 0.3$ with a typical concentration of $\mathrm{Cr} 0.5$ at.\% (i.e. $N_{\mathrm{Cr}}=7.5 \times 10^{19} \mathrm{~cm}^{-3}$ ). We also studied the samples of $\mathrm{Pb}_{1-y} \mathrm{Cr}_{y} \mathrm{Te}(0 \leq y \leq 0.01)$ and $\mathrm{Sn}_{1-y} \mathrm{Cr}_{y} \mathrm{Te}(y=0.005$ and 0.02). The chemical composition of our samples was checked by $X$-ray fluorescent analysis revealing a rather strong effect of distribution of chromium ions along an ingot. The crystals of $\mathrm{PbTe}: \mathrm{Cr}$ and $\mathrm{SnTe}: \mathrm{Cr}$ were also investigated by $X$-ray Debye powder method. They were found free of second phase inclusions with the cubic lattice constants $a_{0}=6.449 \AA$ and $a_{0}=6.314 \AA$ for $\mathrm{Pb}_{0.99} \mathrm{Cr}_{0.01} \mathrm{Te}$ and $\mathrm{Sn}_{0.995} \mathrm{Cr}_{0.005} \mathrm{Te}$, respectively.

We investigated experimentally the transport properties (Hall effect and conductivity) in the temperature range $T=(4-300) \mathrm{K}$ and the low temperature, $T=(1.6-4.2) \mathrm{K}$, ac magnetic susceptibility. The results of our transport measurements of $\mathrm{PbTe}: \mathrm{Cr}$ agree well with the available literature data $[1,2]$ and can

*This work is supported in part by the Committee for Scientific Research under grant nr 204829101. 
be summarized as follows. The samples with small concentration of $\mathrm{Cr}$ are $p$-type. The $n$-type conductivity is observed only for crystals with $N_{\mathrm{Cr}} \geq 1.5 \times 10^{19} \mathrm{~cm}^{-3}$. The increase of the chromium concentration above $N_{C r} \cong 3 \times 10^{19} \mathrm{~cm}^{-3}$ does not change the electron concentration $n \cong 1.3 \times 10^{19} \mathrm{~cm}^{-3}$. It corresponds to Fermi energy $E_{\mathrm{F}} \cong 100 \mathrm{meV}$. It was suggested [1-4] that this behavior results from the process of the selfionization of $\mathrm{Cr}^{2+}$ ions generating the conducting electrons $\left(\mathrm{Cr}^{2+} \Rightarrow \mathrm{Cr}^{3+}+e\right)$. The electron concentration saturates when the Fermi level reaches the energy level of $\mathrm{Cr}^{2+}$ and the selfionization process stops. It is essentially the same mechanism as the one observed e.g. in $\mathrm{HgSe}: \mathrm{Fe}$ [3] and $\mathrm{PbTe}$ :In [4]. The change of the type of conductivity with increasing $\mathrm{Cr}$ concentration is the result of an interplay :etween the concentration of $\mathrm{Cr}^{2+}$ (donor centers) and the concentration of metal vacancies (present in stoichiometrically grown IV-VI crystals and responsible for the $p$-type conductivity). The electrons generated in the process of selfionization of $\mathrm{Cr}^{2+}$ ions have first to fill the empty electron states in the valence band (generated by metal vacancies) and only then the electron states in the conduction band. Our $\mathrm{PbTe}: \mathrm{Cr}$ samples have very high mobilities $\mu \cong 10^{5} \div 10^{6} \mathrm{~cm}^{2} /(\mathrm{V} \mathrm{s})$. With an increasing tin content in the $\mathrm{Pb}_{1-x} \mathrm{Sn}_{x} \mathrm{Te}: 0.5$ at. \% $\mathrm{Cr}$ alloy one observes a decrease of the electron concentration. For approximately $x \geq 0.1$ the as-grown crystals are $p$-type. It is probably caused by the lack of chromium to fill all the empty states in the valence band. The number of these states is directly measured by the concentration of conducting holes in the $\mathrm{PbSnTe}$ matrix and is known to increase rather fast with increasing tin content. For SnTe:Cr crystals one observes usual $p$-type conductivity with very high concentration of carriers $p \cong 10^{21} \mathrm{~cm}^{-3}$ and very low mobility $\mu \cong 100 \mathrm{~cm}^{2} /(\mathrm{V} \mathrm{s})$.

The typical temperature dependence of the magnetic susceptibility of $\mathrm{PbTe}: \mathrm{Cr}$ and $\mathrm{Pb}_{1-x} \mathrm{Sn}_{x} \mathrm{Te}(x \leq 0.2)$ is presented in Fig. 1. The magnetic susceptibility follows the simple Curie law $(\chi \propto 1 / T)$ indicating the presence in the crystal of the localized magnetic moments. The natural candidates for these moments are $3 d^{3} \mathrm{Cr}^{3+}$ ions which are expected to be $S=3 / 2$ spins in the octahedral crystal field of PbTe lattice. A different behavior is observed in SnTe:Cr (see Fig. 2). There is practically no temperature dependence of the magnetic susceptibility of this sample. It indicates the van Vleck type of magnetic behavior which may be attributed to the $3 d^{4}$ states of $\mathrm{Cr}^{2+}$ ions which are expected to have non-magnetic ground state in the octahedral crystal field [5].

One may attempt to interpret the experimental findings presented above in a model assuming the shift of the $\mathrm{Cr}^{2+}$ level from the conduction band in the case of $\mathrm{PbTe}$ and $\mathrm{PbSnTe}$ to the valence band in the case of $\mathrm{SnTe}$. It will induce the decrease of electron concentration and the changes in the relative concentrations of $\mathrm{Cr}^{2+}$ and $\mathrm{Cr}^{3+}$ ions. In SnTe:Cr one would expect the $\mathrm{Cr}^{2+}$ level to be located below the Fermi level of the crystal. Only magnetically and electrically "neutral" $\mathrm{Cr}^{2+}$ ions are expected to be present in this case. In conclusion, we have observed that $\mathrm{Cr}$ acts as a resonant donor both in $\mathrm{PbTe}$ and $\mathrm{Pb}_{1-x} \mathrm{Sn}_{x} \mathrm{Te}$ with small tin content $(x \leq 0.2)$. Magnetic properties of $\mathrm{PbTe}: \mathrm{Cr}$ and $\mathrm{Pb}_{1-x} \mathrm{Sn}_{x} \mathrm{Te}: \mathrm{Cr}$ are typical for Curie paramagnets and are probably due to the spin magnetic moments of $\mathrm{Cr}^{3+}$ ions. The van Vleck type of paramagnetism observed in $\mathrm{SnTe}: \mathrm{Cr}$ is attributed 

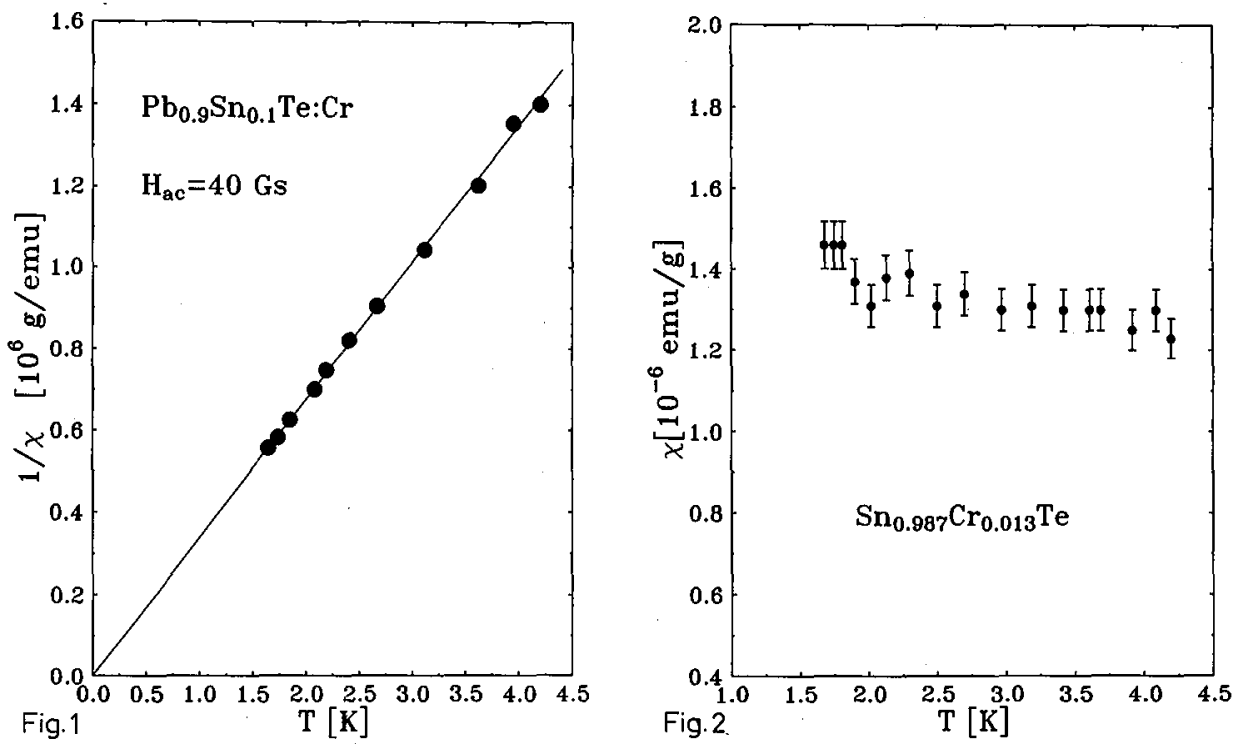

Fig. 1. The temperature dependence of the inverse magnetic susceptibility of the $\mathrm{Pb}_{0.9} \mathrm{Sn}_{0.1}$ Te sample doped with $N_{\mathrm{Cr}} \cong 2 \times 10^{19} \mathrm{~cm}^{-3}$ of chromium. Qualitatively the same behavior is observed for $\mathrm{PbTe}: \mathrm{Cr}$ and $\mathrm{Pb}_{0.8} \mathrm{Sn}_{0.2} \mathrm{Te}: \mathrm{Cr}$ samples.

Fig. 2 The temperature dependence of the magnetic susceptibility of the sample of $\mathrm{Sn}_{0.987} \mathrm{Cr}_{0.013} \mathrm{Te}$. The weak influence of temperature on the magnetic susceptibility and the very small magnitude of it suggest the van Vleck paramagnetism.

to the $\mathrm{Cr}^{2+}$ ions. The magnetic properties of $\mathrm{PbSnTe}$ Cr seem to support the model describing the Cr impurity in IV-VI matrices as a resonant donor with a $\mathrm{Cr}^{2+} / \mathrm{Cr}^{3+}$ state depending on the relative position of the Fermi level and the energy level of $\mathrm{Cr}^{2+}$.

\section{References}

[1] B.A. Akimov, P.V. Verteletskii, V.P. Zlomanov, L.I. Ryabova, O.I. Tananaeva, N.A. Shirokova, Fiz. Tekh. Poluprovodn. 23, 244 (1989).

[2] V.D. Vulchev, L.D. Borisova, S.K. Dimitrova, Phys. Status Solidi A 97, K97 (1986).

[3] for review see; e.g., J. Kossut, W. Dobrowolski, Z. Wilamowski, T. Dietl, K. Świątek, Semicond. Sci. Technol. 5, S260 (1990).

[4] B.I. Kaidanov, Ju.I. Ravich, Usp. Fiz. Nauk 145, 51 (1985).

[5] E.M. Omelyanovskii, V.I. Fistul, Transition Metal Impurities in Semiconductors, Adam Hilger, Bristol and Boston 1986. 